\title{
Las exposiciones temporales como recurso para la clase de ELE
}

\author{
JAUME BRINES GANDÍA \\ Centre d'Idiomes de la Universitat de València \\ jaubri@alumni.uv.es
}

\section{Resumen}

El presente trabajo muestra la rentabilidad que ofrecen los museos, las salas expositivas y el arte en dos ámbitos fundamentales de la docencia de ELE: desde el punto de vista lingüístico y desde el cultural. Las exposiciones museísticas ofrecen múltiples posibilidades cuya adecuada explotación didáctica en el aula puede permitir a los aprendientes de ELE un acercamiento a la lengua y a la competencia intercultural.

Esta actividad práctica se realizó con dos grupos de niveles B1 y B2 en dos exposiciones temporales, sobre Sorolla y Victoria Cano en el Centre del Carme (Valencia). Profesor y alumnos asistieron a una visita guiada de estas exposiciones, en la cual debían completar información relevante en una ficha. Se muestran las ventajas de la integración de los museos y del arte en el aula de ELE, mediante las impresiones de los estudiantes, plasmadas en sus textos escritos tras la visita a estas exposiciones.

Palabras clave: ELE, cultura, exposición, museos

\begin{abstract}
This report shows the profitability that museums, exhibition rooms and art can offer to two different areas in the teaching of Spanish as a foreign language: linguistic and cultural. The museum exhibitions offer multiple resources which, with a good didactic work in the classroom, can provide the students with an approach to the language and the intercultural competence.

This practical activity was carried out in two different groups of B1 and B2 levels, in two different temporary art exhibitions, about Sorolla and Victoria Cano in the Centre del Carmen (Valencia). The teacher and the students attended a guided tour of the exhibitions, in which they had to fill up a sheet of paper with relevant information about the exhibitions. The vantages of arts and the museums integration in Spanish as a foreign language are shown through the students impressions, expressed in their written texts after visiting the exhibitions.
\end{abstract}

Keywords: E/LE, culture, exhibition, museums 


\section{Introducción}

\section{1. ¿Qué es la cultura?}

La cultura en la clase de ELE previo al enfoque comunicativo, ha pasado muchas veces desapercibida y frecuentemente se ha dejado en un lugar secundario la familiarización con los comportamientos, rituales y códigos culturales de la cultura meta (Navarro, 2009:86), pese a constituir un excelente instrumento para la enseñanza de una lengua extranjera y, sobre todo, para que el futuro aprendiente sepa cómo, cuándo y de qué modo usar la lengua. Antes de desarrollar la presente experiencia práctica, es necesario que tratemos el término cultura y las distintas acepciones que supone.

El límite entre la importancia de la lengua y la cultura dentro de una sociedad es muy estrecho, y tanto una como la otra no pueden entenderse por separado. Cualquier profesor de ELE debe tener siempre en cuenta el componente cultural en sus clases. De lo contrario, sus alumnos pueden quedar exentos de un valioso elemento, y sin él no serán capaces de entender por completo la manera de pensar de los nativos, su humor, sarcasmo, dobles sentidos, etc. (Miquel López y Sans Baulenas, 1992: 16).

¿Qué se entiende por cultura? Definir este vocablo es, sin duda, una tarea complicada que no está exenta de polémica.

De las diversas definiciones de cultura para ELE, destaca la propuesta por Miquel López y Sans Baulenas (1992), quienes apuntaban tres tipos de cultura: la cultura a secas, la kultura con $k$ y la Cultura con mayúsculas (Miquel López y Sans Baulenas, 1992: 17). La primera es el conjunto compartido de elementos pautados y no dichos por parte de los individuos de una lengua y cultura, que constituyen un estándar cultural. La kultura se refiere al estrato bajo de la cultura: es la capacidad de identificar social o culturalmente a un interlocutor y actuar lingüísticamente adaptándose a este, como pueda ser el argot juvenil. Por último, la Cultura, para estas autoras, constituye el conjunto de hitos artísticos, como literarios, pictóricos, arquitectónicos, musicales, etc. de cualquier país, siendo el estrato superior de la cultura. Esta es una de las definiciones de cultura más detallada y clarificadora de cuantas haya para ELE.

Así, cualquier extranjero que esté aprendiendo español en España, necesita aprender este tipo de aspectos culturales para entender la realidad de la sociedad que le rodea: cualquiera de los 3 tipos de cultura o al menos uno de ellos. En este sentido, Iglesias Casal (2000) afirma que «comprender el contexto cultural de comunicación es fundamental muchas veces para descodificar con acierto un mensaje». Combinar todos estos conceptos de cultura en el aula de ELE es una ardua tarea y para el profesor puede suponer un reto integrarlos en su clase. Evidentemente, los manuales de lengua incluyen este componente entre sus materiales, aunque presentan algunos inconvenientes, como el hecho de centrarse casi siempre en la Cultura (con mayúsculas) e ignorar sus otras dimensiones, o caer en tópicos y en el etnocentrismo, mostrando una imagen desfasada e irreal. Así, el Instituto Cervantes ofrece en su Plan curricular (2008) toda una serie de elementos y pautas culturales que los aprendientes deben ser capaces de saber o reconocer dependiendo del nivel de competencia de estos.

Actualmente hay infinidad de recursos para poder crear materiales didácticos interesantes a la hora de enseñar cultura. Uno de estos son sin duda los museos, puesto que ofrecen un componente artístico que puede permitir la creación de innumerables actividades centradas en la lengua meta. Por una parte, la imagen puede permitir el 
desarrollo de los alumnos para la creación en ELE de textos, ya sean escritos u orales. Por otra y aunque menos presente en los museos, se encuentran los textos de las cartelas y toda la producción escrita en forma de crítica artística.

El museo y las salas expositivas forman, pues, parte del componente cultural y tienen una serie de características que, bien diseñadas por el profesor, pueden hacer de algunas tareas en el aprendizaje de ELE (como la expresión escrita y la comprensión oral) un elemento dinamizador y distendido en la enseñanza de esta lengua y su cultura. Así, el museo se convierte en:

(...) un nuevo escenario real, con situaciones de comunicación verídicas y que nos servirá de input. Este nuevo contexto es el museo, entendido como lugar de encuentro entre la pedagogía, la didáctica, la cultura y el arte que nos acerca a la realidad de la sociedad española. Los museos vienen mostrándose como espacios para el aprendizaje de contenidos pedagógicos, convirtiéndose en un elemento comunicativo desencadenante del aprendizaje de lenguas extranjeras y del conocimiento del patrimonio cultural de un país (Blanco y Mazoy, 2010: 6). Este acercamiento a la perspectiva patrimonial pretende convertirse en un nuevo producto pedagógico, es decir, una guía-taller que ayude a desarrollar clases de español en el ámbito del Museo.

Gómez López (2015: 205)

Este escenario museístico en el que se dan situaciones reales de lengua, es el que configuró la actividad-experiencia, con el museo-sala expositiva como un espacio de aprendizaje para los estudiantes de E/LE, tal y como apuntaba esta autora.

\subsection{Aprendizaje integrado de Lenguas extranjeras (AICLE)}

La experiencia que se puso en práctica está basada en el Aprendizaje integrado de contenidos y lenguas extranjeras (AICLE) o Content and Language Integrated Learning en inglés (CLIL). Esta metodología consiste en la enseñanza de una materia mediante una lengua meta, con lo cual el estudiante integra la enseñanza de esa asignatura con la L2, en el caso de esta actividad, el español.

El AICLE tiene dos objetivos principales como son el desarrollo por medio de un aprendizaje dual (Marsh 2002:57) de una materia no lingüística y de un idioma, de ahí la integración de contenidos, dando respuesta a objetivos educativos específicos. Así, en opinión de Navés y Muñoz:

El Aprendizaje Integrado de Lenguas Extranjeras y otros Contenidos Curriculares implica estudiar asignaturas como la historia o las ciencias naturales en una lengua distinta de la propia. AICLE resulta muy beneficioso tanto para el aprendizaje de otras lenguas (francés, inglés,...) como para las asignaturas impartidas en dichas lenguas. El énfasis de AICLE en la "resolución de problemas" y "saber hacer cosas" hace que los estudiantes se sientan motivados al poder resolver problemas y hacer cosas incluso en otras lenguas.

Navés y Muñoz (2000: 2)

En lo que respecta a la combinación de esta teoría didáctica con el componente cultural, es interesante la siguiente apreciación:

(...) el término lengua se refiere también a todo un sistema de acción social que conlleva significados culturales. Así la lengua y la cultura se nos presentan como un todo indisociable, 
porque a todo hecho de lengua subyace un hecho de cultura (Guillén, 2004: 838). Los contenidos culturales responden a una estructura que comprende el lenguaje, las artes, las costumbres, los ritos, fiestas, etc.; una estructura que muestra la proyección del hombre en el tiempo

(Gómez López, 2015: 206).

Esta combinación del AICLE con la cultura se llevó a cabo mediante la promoción de una materia, el arte, emparentada directamente con la cultura (en este caso la Cultura en mayúsculas de Miquel y Sans) y la L2, con lo que el Aprendizaje integrado de lenguas tiene un punto en común con esta experiencia: se usa la lengua como herramienta para lograr los objetivos. De ese modo se tratan contenidos artísticos mediante la lengua meta, y así los aprendientes activan la lengua y la competencia intercultural, lo que supone un reto cognitivo para estos al poder expresarse en la L2 y al mismo tiempo experimentar situaciones reales en esta lengua.

\section{Objetivos}

La actividad práctica pretendía alcanzar varios objetivos, destacando la combinación del aprendizaje del E/LE con el de la cultura española (y valenciana por extensión) mediante obras de arte. Por eso la sala expositiva o museo fue el elemento en torno al cual giró la experiencia para que los alumnos desarrollasen sus competencias mediante la visita a este y la visualización de las obras de arte.

En primer lugar, los cuatro objetivos fundamentales que se plantearon fueron los siguientes:

- Atraer la atención del estudiante hacia aspectos culturales

- Fomentar un contexto de aprendizaje distendido

- Despertar el gusto estético a través de un acercamiento al arte

- Desarrollar la expresión escrita y activar otras competencias como la comprensión oral y la lectora-visual

Por ello, el diseño pedagógico de la actividad se centró en la selección de las exposiciones temporales, Apuntes en la Arena, de Joaquín Sorolla y Huellas \& Ecos de Victoria Cano, que estuvieron expuestas en el Centre del Carme de Valencia entre el 2 de febrero y 29 de mayo de 2016 (la primera) y el 11 de febrero al 15 de mayo de 2016 (la segunda). Los alumnos tenían que hacer varios ejercicios en la sala expositiva, pero también en casa, con lo que la visita al museo era obligatoria.

Al final de dicha actividad y con el objeto de conocer las opiniones de los aprendientes acerca de la actividad y sus sensaciones, se debía completar una encuesta para conocer el grado de satisfacción de los estudiantes.

\section{Tipo de alumnado}

La mayoría de participantes eran estudiantes extranjeros en Valencia becados con el programa Erasmus, todos ellos divididos en dos grupos de niveles B1 y B2. La diversidad nacional de los estudiantes era una característica notable, si bien es cierto que el grupo de B1 era un poco más homogéneo. Este grupo estaba compuesto por 14 estudiantes con edades comprendidas entre los 19 y los 27 años, todos ellos disfrutando de la beca Erasmus excepto dos, un estudiante brasileño y otro coreano, con becas 
internacionales de sus países. En cuanto al sexo, de los 14 había una mayoría femenina ya que solo había 4 estudiantes varones. El origen de los alumnos era el siguiente: 4 alemanes ( 3 mujeres y un varón), 3 noruegas, 3 ingleses ( 2 mujeres y un varón), una francesa, una polaca-estadounidense, un brasileño y un surcoreano.

Respecto al grupo de B2, este era más heterogéneo puesto que había seis parejas con las mismas procedencias y el resto era de diversos países, aunque 3 de ellos de la zona de Escandinavia, conformando una clase de 16 personas. La diferencia de edad en este grupo era más notoria dado que 4 de los estudiantes ya eran profesionales y había una doctoranda. Estos cuatro alumnos superaban los 30 años e incluso uno de ellos tenía 45 (el alumno italiano). Los demás se situaban en edades comprendidas entre los 21 y los 29 años y muchos de ellos estaban ya finalizando sus respectivas carreras o incluso estudiando una segunda. Entre las parejas de los mismos países había 2 alemanes (un varón y una mujer), 2 finlandeses (un varón y una mujer), 2 húngaros (un varón y una mujer), 2 inglesas, 2 italianos (un varón y una mujer) y 2 sirios (un matrimonio). El resto eran una brasileña, un danés, una noruega y un sueco.

Los estudiantes eran estudiantes de ELE en el Centro de Idiomas de la Universitat de València (a partir de ahora CDI) y cabe destacar que, gracias a esta experiencia, por primera vez se han realizado visitas guiadas a museos como parte integrante de las clases de ELE. En estas, además de la interacción del profesor/guía con los estudiantes, la interpretación crítica de los espacios museísticos vistos y su plasmación por parte del alumnado, se ha materializado mediante dos de las 4 redacciones que se les exige a lo largo del curso semestral. Estas actividades fueron pensadas por el profesor, comunicadas a la dirección, aceptadas y realizadas en horario extraescolar. Los resultados se muestran a continuación tras una breve descripción metodológica.

\section{Metodología y organización de las actividades}

La actividad cultural en el Centre del Carme de Valencia se efectuó mediante dos ejercicios en dos grupos de B1 y B2 de ELE. De esta manera, se llevó a cabo una visita museística por cada grupo en horario extraescolar. Ambas visitas contaron con la participación de una mediadora cultural (una diferente por visita), que hizo la visita guiada de la exposición de Joaquín Sorolla, Apuntes en la arena.

Si bien la idea de hacer las visitas en viernes evitaba el solapamiento de la mayoría de las clases universitarias de los alumnos, algunos plantearon impedimentos para asistir argumentando excursiones y viajes por la llegada de familiares, ante lo cual se les trató de facilitar la asistencia con la visita del otro grupo. Además, algunos estudiantes fueron acompañados por amigos no pertenecientes al centro y unos pocos fueron solos a los museos por su cuenta, sin las explicaciones del profesor o de las mediadoras culturales.

En cuanto al aprovechamiento didáctico de las visitas, se plantearon dos actividades que junto con la visualización de las salas posibilitaran el desarrollo de varias destrezas comunicativas. Por una parte, la simple asistencia ya permitió que los estudiantes practicaran su destreza de comprensión oral al escuchar a la mediadora cultural y a su profesor. En ambas visitas se preguntó al alumnado diferentes cuestiones relativas a las exposiciones y en algunos casos se llegaron a crear pequeños debates a partir de los diferentes puntos de vista de los estudiantes. Por otra parte, en ambas salidas había de completarse una ficha diseñada por el profesor, relativa a cada exposición, con 
elementos explicados a lo largo de la visita (léxico), otros en los que el estudiante debía comentar elementos que le hubiesen gustado y los interpretara, y otros elegidos por el docente para ser analizados por los alumnos mediante la elaboración de pequeños textos escritos en ambos casos. De este modo, la ficha hacía especial hincapié en la práctica y el desarrollo de la expresión escrita, con la redacción como tarea final. Finalmente, la última parte de la ficha consistía en una pequeña encuesta relativa al museo y sus exposiciones, para establecer un criterio de satisfacción y conocer la opinión del alumnado.

\section{Equipo técnico}

El equipo que compuso la secuenciación cultural tuvo 3 protagonistas. En primer lugar, el profesor fue el que diseñó y planificó las actividades. En segundo lugar, el profesor contó con la ayuda de dos estudiantes del Diploma de Educación artística y gestión de museos de la Universitat de València, quienes muy amablemente no solo aportaron ideas y sugerencias en el diseño de la actividad, sino que se prestaron a realizar la visita guiada a la exposición de Sorolla, tal y como venían haciendo durante su periodo de prácticas en el Centre del Carme. Su participación y protagonismo fue esencial para que algunos alumnos se tomaran más en serio la actividad al no ser el profesor el único elemento mediador y acceder así de primera mano a las explicaciones de unas profesionales dedicadas de pleno a su actividad como mediadoras culturales.

En tercer lugar y protagonistas principales, los estudiantes, divididos en dos grupos con los mismos horarios a lo largo del segundo semestre, entre mediados de febrero y junio, dos días a la semana por grupo.

\section{Integración de la actividad en la expresión escrita}

La actividad pasó a formar parte de la nota de expresión escrita, en su apartado de redacciones como ejercicios para casa. A lo largo del curso los alumnos deben entregar 4 redacciones diferentes que son corregidas por el docente, para de ese modo ir practicando y mejorando esta destreza. El hecho de integrar la actividad como parte de la calificación del curso tenía un doble objetivo: por un lado, al darle ese carácter obligatorio, se forzaba en cierta manera a la participación del alumnado en las salidas museísticas y por otro, se le dotaba de un aspecto de oficialidad con respecto al CDI. Es interesante constatar que ambas visitas y actividades fueron bastante bien acogidas por los estudiantes, especialmente por el grupo de B2.

La expresión escrita es una competencia con escasa presencia en las clases y en los materiales de ELE (Cassany, 1999: 47). Ello es debido como decían González Sainz y Artuñedo Guillén (1995: 169), a «que el tratamiento de la expresión escrita en los métodos tradicionales se centra en el texto (producción del alumno) como pretexto: tal es el carácter de ejercicios como los temas de redacción, comentario y los dictados».

Así, la redacción siempre ha destacado por ser un ejercicio escrito dominante, con un objetivo puramente gramatical para la asimilación por parte del alumno de diversas tipologías textuales (González Sainz y Artuñedo Guillén, 1995: 169). Un ejercicio tipo suele ser contar rutinas actuales para el correcto uso del presente, rutinas en pasado para el correcto uso del imperfecto y contar un viaje para la combinación de los verbos de 
pasado. En muchas ocasiones, este tipo de ejercicios producen como destacan González Sainz y Artuñedo Guillén (1995:169) «"blancos" de imaginación, se bloquea ante la obligación de crear, de ser original sobre el papel y recurre a estereotipos interiorizados que dejan bastante que desear en cuanto al desarrollo de la creatividad».

Por desgracia, la escritura ha sido usada como una forma de evaluación de contenidos gramaticales, o como un "falso" ejercicio creativo de expresión de la opinión personal (González Sainz y Artuñedo Guillén, 1995: 169). Sin embargo, la expresión escrita, según Cassany (1999: 50) también puede ser concebida como herramienta de adquisición lingüística. Esta puede ser un medio para el aprendizaje de ELE, destacando diversas funciones como la «registrativa (anotar léxico y expresiones desconocidas, copiar citas), manipulativa (resúmenes y esquemas de textos comprendidos, guiones para producciones) o incluso epistémica (elaboración de opiniones, análisis gramaticales y discursivos)»

Cassany también distingue ciertas particularidades, según el objeto específico de aprendizaje:

2.1. DESARROLLO DE LAS HABILIDADES ORALES. La escritura registrativa se convierte en recurso mnemotécnico para recordar ítems lingüísticos que se escucharon y/o se deberán pronunciar.

2.2. APOYO AL DESARROLLO DE LA GRAMÁTICA. La escritura manipulativa y epistémica permite analizar el sistema lingüístico de la lengua meta e incrementar el conocimiento gramatical del aprendiente.

2.3. TRANSMITIR CONTENIDOS SOCIOCULTURALES. La producción manipulativa y epistémica de escritos también permite trabajar temas culturales. Por ejemplo, resumir una lectura o redactar una carta con opiniones personales sobre la cultura de la lengua meta o la L1 de origen, permiten incrementar la conciencia sobre diferencias socioculturales entre las comunidades lingüísticas de cada idioma.

Cassany (1999: 50)

En esta actividad de museos, se intentó combinar todas estas particularidades descritas por Cassany (1999:50). El desarrollo de habilidades orales se encontraría con la actividad de léxico de la visita guiada a la exposición de Sorolla, con términos relativos a la obra de este pintor, explicados por las mediadoras culturales. El apoyo al desarrollo de la gramática se centraría en la producción de pequeños textos de opinión, referidos a las obras vistas en ambas exposiciones. Por último, el hecho de la propia actividad ya conlleva una transmisión implícita de contenidos socioculturales y del desarrollo de la competencia intercultural, puesto que cada uno de los estudiantes pudo ver una obra de la cultura meta y compararla con la de origen.

La actividad pretendió aunar todos estos aspectos, dando mayor importancia a la competencia de la expresión escrita, pero utilizando otras tales como la comprensión oral, mediante la audición de las mediadoras culturales y el profesor y la lectora-visual, a través de los cuadros.

\section{Resultados de la actividad}

Las visitas al Centre del Carme se llevaron a cabo en dos fechas para que los alumnos que no pudieran asistir a una de las fechas, tuvieran una alternativa aprovechando que 
en el Centre del Carme había otra exposición, la de Ecos \& Huellas de Victoria Cano, la actividad se extendió también a esta. Así la visita guiada inicial de la exposición de Sorolla, Apuntes en la arena, contrastaba con la de la artista valenciana contemporánea, además de ser esta semiguiada y un poco más libre. La obra de Sorolla permitió a los alumnos ver una Valencia decimonónica, donde los que frecuentaban la playa no eran la mayoría de la población, sino los pescadores y sus niños, que trabajaban en la mar y tenían la piel curtida. La exposición de Victoria Cano también hizo reflexionar a los grupos en cuanto a las trazas que los humanos dejamos en nuestro entorno y cómo podemos maltratar la naturaleza y el impacto que nuestras acciones tiene sobre ella.

Antes de iniciar la visita por las salas expositivas, el profesor entregó a los alumnos el documento con las actividades que estos debían completar. Una vez en el interior del edificio, a medida que las mediadoras culturales llevaban a cabo su cometido, los alumnos debían ir cumplimentando este documento. La primera cara de este se centraba en la obra de Sorolla, la segunda en la de Victoria Cano y el final de esta era una encuesta de satisfacción. En ambos casos, se leyeron las preguntas antes de entrar en el centro, para aclararlas en caso de no ser bien comprendidas por los estudiantes.

La primera parte de la ficha, consistía en esta pregunta:

1. Podrías definir estos conceptos de la exposición de Sorolla. ¿Por qué son importantes en su obra y en esta exposición? ¿Qué te sugieren?

a. Esbozo; b. Apuntes; c. Movimiento; d. Plein Air; e. Fugaz; f. Pesca del bou.

Es curioso que la mayoría de alumnos confundieron los conceptos esbozo y apuntes, a pesar de las explicaciones claras de las mediadoras. Esta parte de léxico era la única que debían ejecutar los alumnos durante la visita a las exposiciones.

La pregunta relacionada con la exposición de Sorolla era la siguiente:

¿Te ha gustado o no, la exposición? ¿Qué emociones te despiertan los cuadros de Sorolla? ¿Qué destacarías de los cuadros que has visto? ¿Qué elementos de sus cuadros ves reflejados de la vida de Valencia? Desarrolla estas ideas.

Las respuestas fueron múltiples pero la mayoría incidía en la vida costumbrista del siglo XIX, la luz de la ciudad y el contraste de la vida de los trabajadores de la playa con las escenas de la burguesía en los cuadros de la última sala. Estos conceptos fueron ampliamente descritos por las mediadoras, que además a lo largo de las visitas iban acompañadas de un carro con diversos materiales como vinilos, cartas, mapas, etc. para explicar conceptos de la obra de Sorolla, tanto a nivel técnico (colores, luz, pinceladas, uso del carboncillo, etc.) como figurativo (personajes, paisajes, escenas, objetos, etc.).

Tras la visita de esta exposición, los alumnos fueron con el profesor y las mediadoras culturales a la sala Ferreres, donde se localizaba la exposición de Victoria Cano Ecos \& Huellas. Aquí la visita fue semiguiada ya que tanto el profesor como las mediadoras hicieron alguna interpretación con respecto a la obra expuesta, pero siendo más libre. De hecho, se formaron pequeños grupos y en algún caso los estudiantes iban solos. En esta exposición, las preguntas que se planteaban a los alumnos eran las siguientes:

La obra de V. Cano habla sobre la constante transformación de la naturaleza y de la compleja realidad en la que vivimos. ¿En qué has podido percibir estos elementos en la exposición y qué sentimientos te ha provocado? 
De las obras que has visto de Victoria Cano, ¿cuál te ha llamado más la atención? ¿Por qué? ¿Y la que menos te ha gustado? ¿Qué te ha transmitido esta exposición?

Si bien es cierto que estas preguntas condicionaban de alguna manera las respuestas por estar dirigidas, las apreciaciones de los estudiantes fueron bastante interesantes, ya que en la mayoría de los casos se interpretaron las obras con el título de la exposición. Los alumnos consideraron la obra de la artista y la relacionaron con problemas actuales que afectan a nuestro planeta. Así, en varias de las redacciones se exponía que los seres humanos dejamos una huella en nuestro entorno y por lo tanto en la naturaleza y los espacios que habitamos. A continuación, se muestran dos ejemplos:

\begin{abstract}
Alumna italiana (B2)
La exposición de Cano pone el foco en las transformaciones provocadas por el ser humano, pero también sus posibilidades expresivas: la naturaleza reproducida por Cano está toda pervadida por contrastes de color que me han llamado la atención porque delinean perfiles humanos en todas partes. Las pinturas de Cano me han gustado mucho por este elemento de inserción del hombre en paisajes naturales, opuesto a las contradicciones profundas en las cuales vivimos en la época de la modernidad. El objeto del arte que se explica en sus obras es la reflexión acerca de nuestros objetivos: ¿el desarrollo humano está siguiendo el camino correcto?
\end{abstract}

\begin{abstract}
Alumna polaco-estadounidense (B1)
Me gustó las obras de Victoria Cano. Su propósito era claro. La transformación y la complejidad esta visible. Para mí, he apreciado este literalmente y figurativamente. Nosotros dejamos nuestra huella permanente en el presente. Debemos tratar de apreciar la naturaleza más y vivir en armonía.
\end{abstract}

Finalmente, los alumnos completaron la última parte de la ficha, la que se centraba en la encuesta de satisfacción. Respecto a las notas que otorgaron a las exposiciones, parece que la de Victoria Cano gustó más que la de Sorolla, si bien el grupo de B2 otorgó el mismo resultado, 8/10, a ambas exposiciones, mientras el grupo de B1, dio un 6,5/10 a la de Sorolla y también un 8/10 a la de Cano.

De alguna manera, las respuestas que elaboraron supusieron una pequeña práctica de realidad, ya que en cierto modo los estudiantes se transformaron en críticos de arte y plasmaron las emociones que experimentaron al observar ambas exposiciones. A continuación, se muestran tres fragmentos representativos de las composiciones de los alumnos, en los cuales no solo realizaron interpretaciones personales de las exposiciones, sino que también demostraron entender el discurso explicado por las mediadoras culturales (véase el comentario del alumno danés):

\title{
Alumna noruega (B1)
}

Lo me gustó mucho, a mi me gusta ir a ver exposiciones y también me encanta el arte en general. Era muy bien de ver a los cuadros de un artisto valenciano del pasado, porque podía ver como era la cultura. Como que mucha gente trabajaba a la playa y con los barcos. Y también la diferencia entre la gente rica y los trabadores.

\footnotetext{
Alumno danés (B2)

Lo encontré bastante interesante que la exposición mostró los esbozos de Sorolla puesto que ellos revelan el trabajo 'detrás' de la obra final y, normalmente, son algo que no se ve. Sin embargo, me gustaron más los cuadros acabados ya que ellos me despertaron más emociones y pensamientos.
}

Alumno italiano (B2) 
Victoria Cano, con su creatividad y sus nuevas formas de expresión, desde las más tradicionales como la pintura, hasta la aplicación de las tres dimensiones al arte, ha llamado mucho mi atención por su forma de mostrar el mar Mediterráneo. Hablando de la inmigración, lo representa como un mar cerrado, rodeado de alambrada. Sin duda, ésa tiene también el significado de que muchos países no quieren enfrentarse al problema de los miles de personas que han muerto. Además, otras naciones (Italia incluida) lo administran muy mal

Como suele suceder en estos niveles, uno de los errores gramaticales más repetidos fue el mal uso del imperfecto y del pretérito indefinido. Sin embargo, más allá de estos errores gramaticales, lo más llamativo y enriquecedor de esta experiencia fue que los estudiantes realizaron críticas discursivas escritas con el género del diario (opiniones ante situaciones vividas) con una aproximación al de la crítica artística, puesto que comentaban las obras de arte de las exposiciones que presenciaron.

\section{Conclusiones}

El trabajo aquí expuesto muestra lo que fueron las dos visitas museísticas que los grupos de B1 y B2 llevaron a cabo. Estas, con sus respectivas redacciones, tuvieron como consecuencia diferentes logros en lo que a los estudiantes se refiere. Por una parte, es destacable el acercamiento de los estudiantes a los museos, centros contenedores de cultura y que en la mayoría de casos no formaban parte de sus recorridos habituales (discotecas y pubs de la ciudad). Por otro lado, también se les acercaba a dos realidades artísticas que forman parte de la ciudad de Valencia: la pintura costumbrista de Sorolla (s. XIX y principios del s. XX) y el arte contemporáneo de la ciudad, representado por la obra de Victoria Cano (siglo XX y principios del XXI).

Mediante las actividades, la formación de ELE adquirió en ambos grupos un componente cultural y, por qué no decirlo, intercultural, puesto que en algunos casos surgieron debates interesantes en torno a las diferentes realidades culturales de los alumnos y su comparación con la valenciano-española. Las visitas además ayudaron a deslocalizar del aula la clase y sumergir a los estudiantes en un entorno más informal, como son los espacios museísticos. En ese sentido los estudiantes quedaron muy satisfechos con ambas mediadoras, que además hicieron participar a los estudiantes mediante preguntas y con la lectura de cartas escritas por Sorolla a su mujer Clotilde, con lo que en parte se hizo una práctica de expresión oral.

Con todos estos elementos se consiguió incluir el ELE en el ámbito de la educación artística, lo que posibilitó tal y como se observó en algunas de las redacciones de los estudiantes, una concienciación y de manera inesperada, pero como consecuencia de esta, una educación en valores a través del arte. Este sorprendente punto se hizo patente en algunas de las respuestas sobre ambos artistas, especialmente en la toma de conciencia de algunos alumnos con los problemas actuales reflejados en la obra de Cano. Se consiguió, mediante estas visitas, cohesionar los grupos puesto que se conectó el aprendizaje del ELE a la dimensión emotivo-afectiva y reelaboraron discursos previos inicialmente diferentes. Así el arte y las exposiciones se convirtieron en un espacio de unión entre los estudiantes y el profesor que les permitió a todos mejorar y aprender los unos de los otros.

Estas visitas deberían haber tenido una proyección también en el aula, para generar más debate y escuchar de primera mano las opiniones de todos los alumnos. Queda 
pendiente esta tarea por parte del profesor, ya que es consciente de que las actividades podrían haber sido tratadas también en el aula, pero la falta de tiempo fue fundamental en este sentido.

\section{Bibliografía}

CASSANY, D. (1999). «La composición escrita en E/LE». Expolingua, 9, 47-66.

GonzÁlez SÁinz T. y Artuñedo Guillen, B. (1995). Propuestas didácticas para la expresión escrita en clase de ele. Recuperado el 21/4/17, de: http://cvc.cervantes.es/ensenanza/biblioteca_ele/asele/pdf/06/06_0168.pdf

Iglesias CAsal, I. (2000). Diversidad cultural en el aula de E/Le: La interculturalidad como desafio y como provocación. Recuperado el 24/4/17, de: http://pendientedemigracion.ucm.es/info/especulo/ele/intercul.html

Instituto Cervantes (2008). Plan curricular del instituto cervantes. Niveles de referencia para el español. Madrid: Biblioteca nueva.

López Gómez, E. (2015). «Entre temples y claroscuros. Una propuesta didáctica de Aprendizaje Integrado de Contenidos y Lenguas Extranjeras». Foro de Profesores de $E / L E, 11,205-214$.

Martínez Arbelaiz, A. (2002). El componente cultural en los cursos de español como L2: una propuesta de clase fuera de clase. Recuperado el 15/6/16, de: http://cvc.cervantes.es/ensenanza/biblioteca_ele/asele/pdf/13/13_0586.pdf.

MiQuel, L. y N. SANS (1992). «El componente cultural: un ingrediente más en las clases de lengua, Cable, $9,15-21$.

Navarro Serrano, P. (2009). «Cultura con eñe: Cultura, sociocultura e intercultura en la clase de ELE». Tinkuy, 11, 8-93.

Marsh, D. (2002). CLIL/EMILE. The European Dimension. Actions, Trends and Foresight Potential. Jyväskylä: DG EAC, European Commission.

NAVÉS, M.T. y MuÑOZ, C. (2000). «Usar las lenguas para aprender y aprender a usar las lenguas extranjeras. Una introducción a AICLE para madres, padres y jóvenes». En MARSh, D. y LANGÉ, G. (eds.). Using Languages to Learn and Learning to Use Languages. (pp. 1-16 parte 3). Jyväskylä: UniCOM. 